\title{
High-Resolution Ultrasonography Diagnosis of Meniscal Cyst from Lateral Meniscus
}

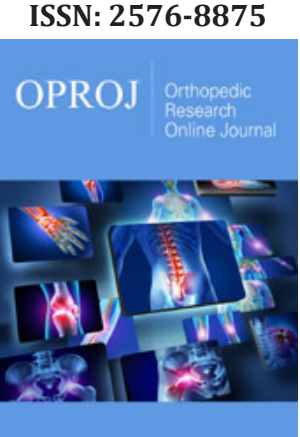

*Corresponding author: Rajul Rastogi, Teerthanker Mahaveer Medical College and Research Center, Moradabad, Uttar Pradesh, India

Submission: 監 May 19, 2020

Published: 眥June 02, 2020

Volume 7 - Issue 2

How to cite this article: Rajul Rastogi, Yuktika Gupta, Neha, Gulzari Lal Meena, Vijai Pratap. High-Resolution Ultrasonography Diagnosis of Meniscal Cyst from Lateral Meniscus. Ortho Res Online J. 7(2). OPROJ. 000656. 2020. DOI: 10.31031/OPROJ.2020.07.000656

Copyright@: Rajul Rastogi, This article is distributed under the terms of the Creative Commons Attribution 4.0 International License, which permits unrestricted use and redistribution provided that the original author and source are credited.

\section{Rajul Rastogi ${ }^{1}$, Yuktika Gupta ${ }^{1}$, Neha ${ }^{1}$, Gulzari Lal Meena ${ }^{2}$ and Vijai Pratap ${ }^{1}$}

${ }^{1}$ Department of Radiodiagnosis, Teerthanker Mahaveer Medical College \& Research Center, Moradabad, Uttar Pradesh, India

${ }^{2}$ Department of Radiodiagnosis, SN Medical College and Hospital, Jodhpur, Rajasthan, India

\section{Abstract}

Meniscal cyst is a rare pathology that may present clinically with pain or mechanical symptoms in and around the knee joint. It results from encysted \& extruded synovial fluid through meniscal tear usually secondary to trauma. It is best delineated on magnetic resonance imaging of knee joint which provides a noninvasive diagnosis but is non-therapeutic. However, with the availability of ultramodern ultrasound scanner, it is now possible to diagnose meniscal cyst accurately by high-resolution ultrasonography which also provides a possibility of therapeutic procedure. Hence in this article, we try to emphasize the diagnostic value of high-resolution ultrasonography in rare meniscal cyst pathology of knee joint

Keywords: High-resolution; Ultrasonography; Meniscal cyst; Lateral meniscus

\section{Introduction}

Meniscal cyst (MC) is a rare pathology of knee joint with an estimated incidence of nearly $4 \%$ (range 1-8\%) in previous studies on magnetic resonance imaging (MRI) of knee joint [1,2]. Majority of these cysts are believed to have arisen from the extruded synovial fluid through meniscal tears [1]. These cysts can be intrameniscal (within the substance of meniscus - IMC) or parameniscal (when the cyst crosses the articular margins - PMC) in location [3].

Meniscal cyst usually presents as a focal knee pain or tender palpable swelling but rarely as painless swelling [3]. Due to its common association with meniscal tears, MC may also present with signs of joint stiffness or locking. PMC may be palpable with PMC of Lateral Meniscus (LM) more commonly palpable than that of Medial Meniscus (MM) [4].

Imaging forms the mainstay of diagnosis and its subsequent management. Magnetic Resonance Imaging (MRI) is gold standard imaging tool for evaluation of internal knee joint pathologies including meniscal tears and MC [4]. With the advent of ultramodern ultrasound scanners, high-resolution ultra sonography (HRUS) has assumed the role of a screening or diagnostic imaging tool in variety of pathologies of joints including knee joint. HRUS can sensitively detect all cysts including MC, besides its ability to guide therapeutic aspiration of these cysts in the same sitting [3]. In this article, we present a case of meniscal cyst that was diagnosed correctly by HRUS.

\section{Case Report}

A 26-year old female with focal painful swelling on the antero lateral aspect of knee joint for last few months visited our department for high-resolution ultra sonography to determine the nature of lesion. The swelling was pea-sized and tender without external signs of inflammation. History of trauma was denied. There was no other significant associated clinical finding in the corresponding knee joint. The laboratory findings and radiograph of knee joint were also unremarkable.

HRUS revealed a complex cystic lesion at the site of palpable tender swelling measuring approximately up to $28^{*} 19 \mathrm{~mm}$ showing intense posterior acoustic enhancement (Figure 1). The lesion was located adjacent to lateral meniscus beyond the articular line \& limited externally by lateral collateral ligament. The cyst had smooth walls, echogenic internal debris 
with fine septations and echogenic, punctate, mural nodules. No obvious extension of the cyst into joint cavity was noted. On color doppler imaging few vascular channels were noted in the wall \& septa of the lesion. No evidence of any obvious synovial fluid is noted in the suprapatellar bursa. Based on HRUS findings, a diagnosis of inflamed parameniscal cyst of lateral meniscus was made and patient was advised aspiration and injection of steroids. However, the patient refused and insisted on further investigation.

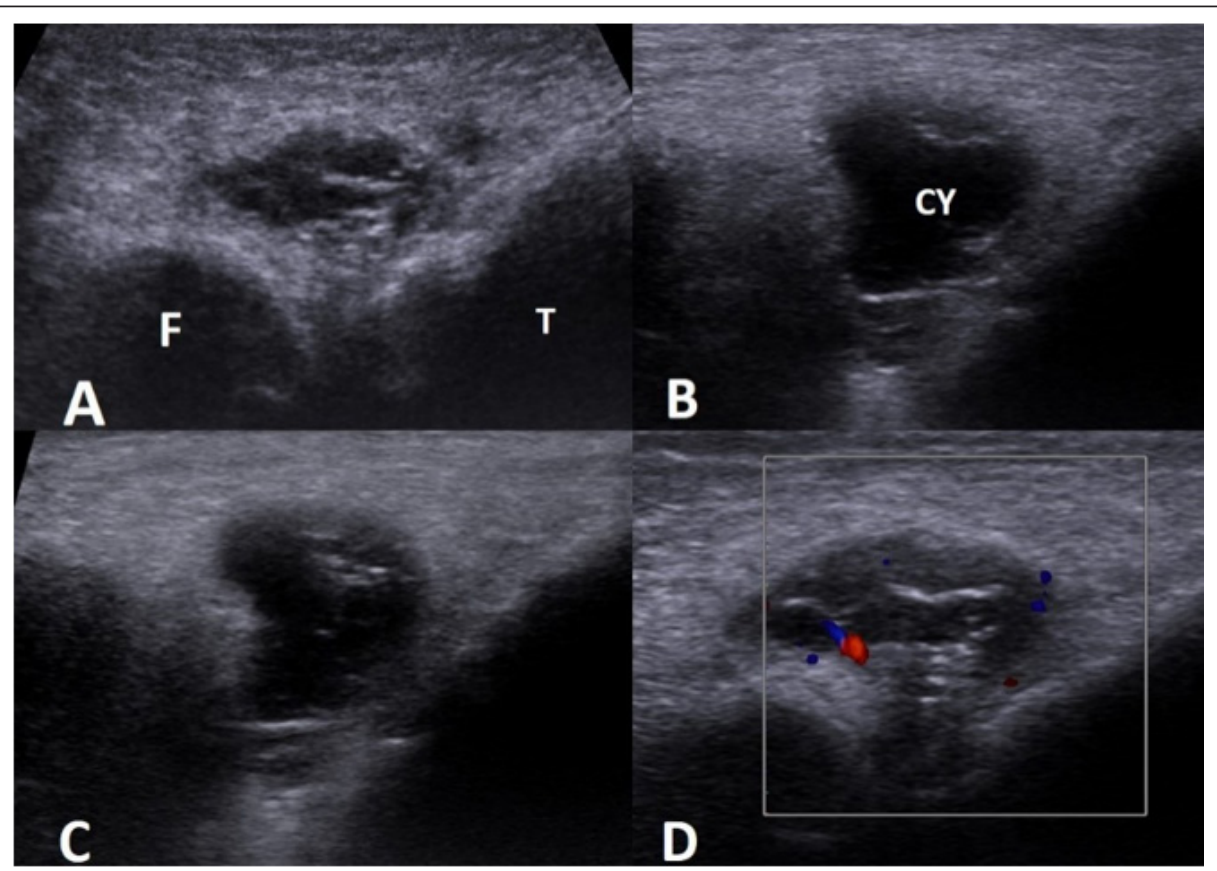

Figure 1: Series of HRUS images (A-C) showing a complex parameniscal cyst adjacent to lateral meniscus with internal septations, echogenic debris and mural nodules as well as subtle vascularity on color doppler image (D) [F: Femoral condyle, T: Tibial Condyle].

MRI of corresponding knee joint was done as part of further work-up and it confirmed the HRUS findings with parameniscal cyst of lateral meniscus appearing intermediate-intensity on T1weighted images and variably hyperintense on T2-weighted, STIR \& fat-suppressed PD images showing significant mural \& septal enhancement on postcontrast, fat-saturated, T1GRE images (Figure
2 \& 3). There was no sign of lateral meniscal tear or joint effusion. No finding was noted in addition to that of HRUS. Following MRI knee joint patient was given treatment options of aspiration with steroid injection or arthroscopic partial resection of lateral meniscus. But patient refused and lost to follow-up.

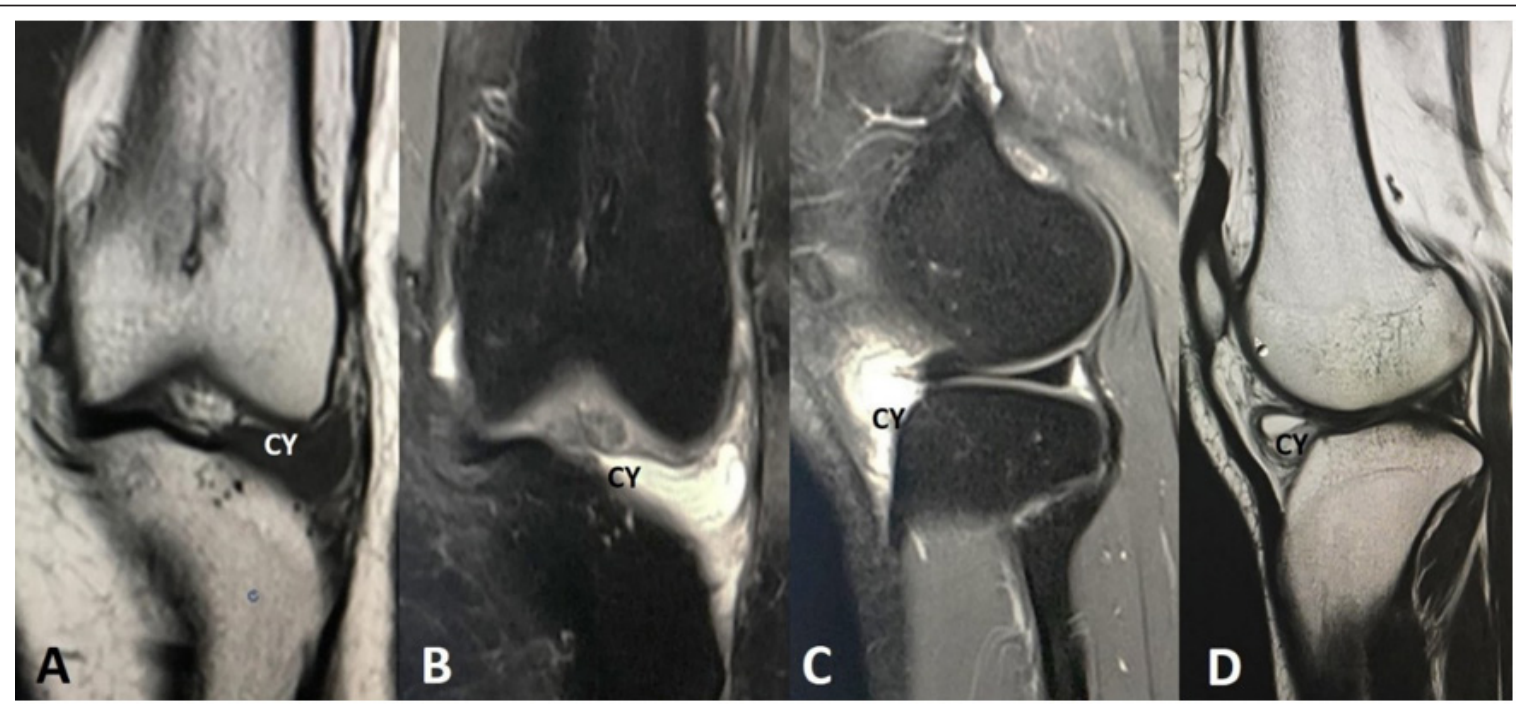

Figure 2: Coronal T1W (A), Coronal STIR (B), Sagittal FS-PD (C) and Sagittal T2W images shows a complex parameniscal cyst of lateral meniscus with thick-walls and internal septations. No evidence of any corresponding meniscal tear or joint effusion was noted [CY: cyst]. 


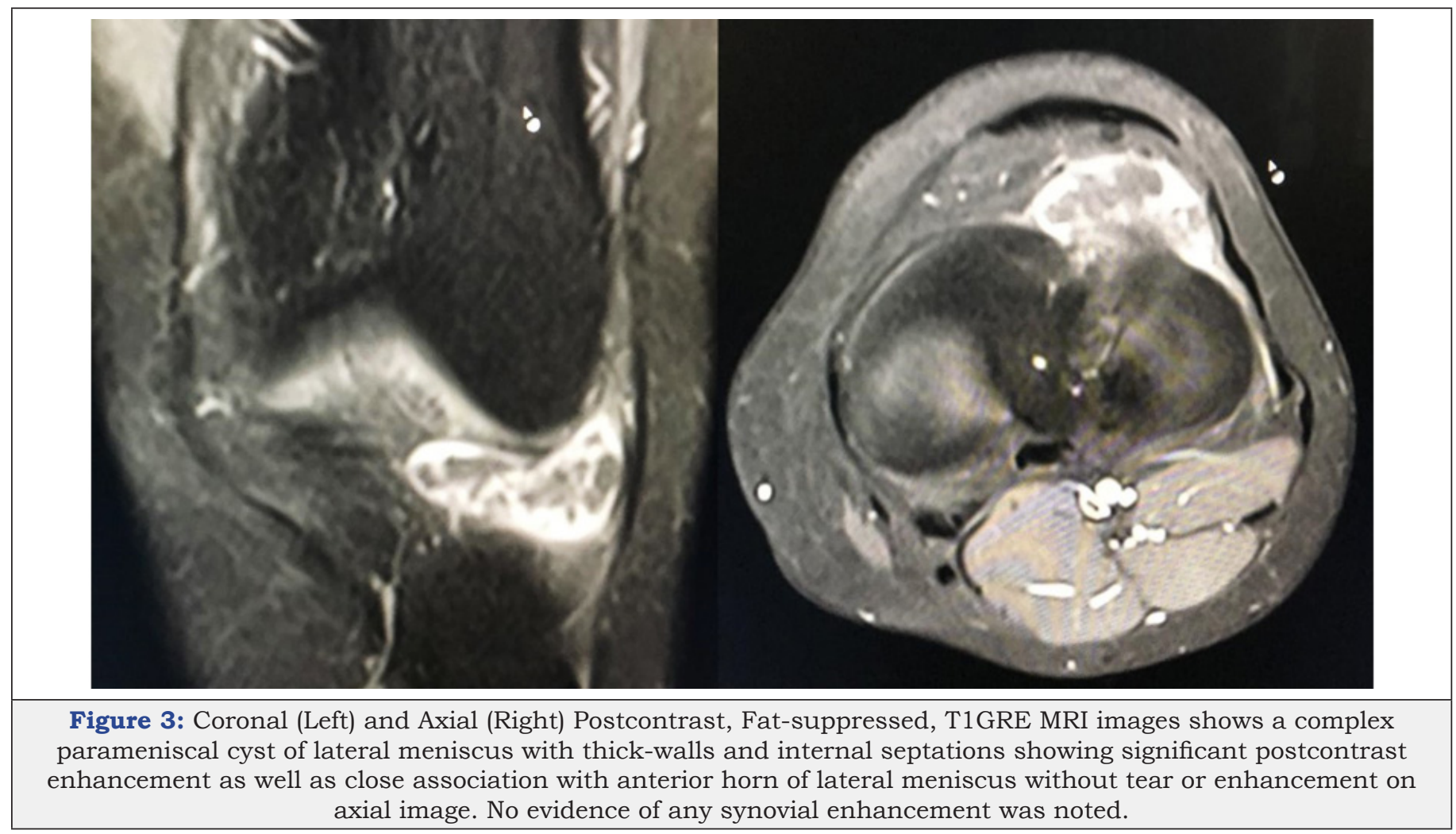

\section{Discussion}

Though MC around knee joint is rare yet when present they are commonly symptomatic either due to pain or mechanical symptoms [3]. Since they are commonly associated with trauma hence loculated extrusion of synovial fluid through the meniscal tear is considered as a major etiopathogenetic mechanism except when related to anterior horn of LM $[1,5,6]$. Few studies have revealed that $\mathrm{PMC}$ adjacent to anterior horn of lateral meniscus are less often associated with meniscal tear partially due to cysts or ganglion of anterior cruciate ligament (ACL) $[5,6]$. Though this did not appear to be in our index case as the epicenter of the cyst was far away from the tibial insertion of ACL.

IMC are usually detected incidentally on knee joint MRI while PMC usually present as painful palpable swelling \& rarely as symptomless swelling [3]. PMC are more commonly associated with LM [4]. HRUS offer the advantages of being a noninvasive, radiation-free \& inexpensive imaging tool to assess any amenable soft tissue swelling including the area around the joints. It offers the advantage of fast \& immediate comparison with corresponding area on opposite side of midline besides being reproducible, easily repeatable and time efficient. Besides, HRUS can be the only imaging tool in patients with contraindication to MRI [3]. Though there are fewer studies on the utility of HRUS in MC yet the conducted studies reveal high sensitivity and specificity. In a study by Rutten et al. [7], HRUS had sensitivity, specificity, Positive Predictive Value (PPV), Negative Predictive Value (NPV) and accuracy of 97\%, 86\%, 94\%, $92 \%$ and $94 \%$ respectively in detection of MC [7]. Another study done by Sorrentino et al yielded a sensitivity, specificity, PPV \& NPV of $94 \%, 100 \%, 100 \% \& 94.5 \%$ respectively in detection of MC by HRUS [8].

On HRUS, MC is seen as thin or thick-walled, cystic lesion with posterior acoustic enhancement (confirming its fluidic nature) within or adjacent to meniscus. The cyst may be anechoic or may show presence of internal debris or septations in cases of chronic / complicated cysts. Inflamed MC may reveal some vascularity on color doppler imaging as seen in our index case explain the pain \& tenderness of the palpable swelling.

HRUS can be used to guide for aspiration of MC including PMC as a conservative method of management followed by injection of corticosteroids $[3,9,10]$. This method has been suggested to complete cure the symptoms in nearly $80 \%$ with long-term resolution in rest in one of the previously conducted study $[3,10]$. In another study on lateral meniscal cyst management, partial lateral meniscectomy along with percutaneous decompressive needling of the parameniscal cyst revealed positive clinical and radiological long-term results without signs of degenerative changes [11].

MRI on the other hand, though expensive \& has limited availability yet it is considered as the goldstandard imaging tool for diagnosis of MC as it not only delineates its extent but also detect other incidental knee joint abnormalities [4]. However, in our case, HRUS was able to delineate the entire extent and complete nature of the PMC without any additional information on MRI knee joint. MRI is especially useful in large or giant PMC with erosion of adjacent bone, dissection into adjacent tissues and superadded infection as STIR images are extremely sensitive to marrow edema 
\& post contrast images detect breach in blood barrier [12,13]. MRI however, is severely limited by the cost of its repeatability.

\section{Conclusion}

Though MRI is the gold-standard modality for imaging meniscal cyst yet HRUS in expert hand approaches the accuracy of MRI, besides providing avenues for therapeutic procedure that is well tolerated and effective in majority of patients.

\section{References}

1. Campbell SE, Sanders TG, Morrison WB (2001) MR imaging of meniscal cysts: incidence, location, and clinical significance. AJR 177(2): 409-413.

2. Anderson JJ, Connor GF, Helms CA (2010) New observations on meniscal cysts. Skeletal Radiology 39(12): 1187-1191.

3. Chen H (2015) Diagnosis and treatment of a lateral meniscal cyst with musculoskeletal ultrasound. Case Reports in Orthopedics 2015: 1-4.

4. Hulet C, Souquet D, Alexandre P, Locker B, Beguin J, et al. (2004) Arthroscopic treatment of 105 lateral meniscal cysts with 5-year average follow-up. Arthroscopy 20(8): 831-836.

5. England E, Wissman RD, Mehta K, Burch M, Kaiser A, et al. (2015) Cysts of the anterior horn lateral meniscus and the ACL: is there a relationship? Skeletal Radiol 44(3): 369-373.
6. De Smet AA, Graf BK, del Rio AM (2011) Association of parameniscal cysts with underlying meniscal tears as identified on MRI and arthroscopy. AJR 196: W180-W186.

7. Rutten MJCM, Collins JMP, van Kampen A, Jager GJ (1998) Meniscal cysts: detection with high-resolution sonography. AJR 171(2): 491-496.

8. Sorrentino F, Iovane A, Nicosia A, Vaccari A, Candela F, et al. (2007) Highresolution ultrasonography (HRUS) of the meniscal cyst of the knee: our experience. Radiologia Medica 112(5): 732-739.

9. Muddu BN, Barrie JL, Morris MA (1992) Aspiration and injection of meniscal cysts. J Bone Joint Surg Br 74(4): 627-628.

10. MacMahon PJ, Brennan DD, Duke D, Forde S, Eustace SJ (2007) Ultrasound-guided percutaneous drainage of meniscal cysts: preliminary clinical experience. Clinical Radiology 62(7): 683-687.

11. Iorio R, Mazza D, Drogo P, Massafra C, Viglietta E, et al. (2020) Lateral meniscal cysts: long-term clinical and radiological results of a partial meniscectomy and percutaneous decompressive needling. Int Orthop 44(3): 487-493.

12. Shin JS, Ou-Yang DC, Gioe TJ (2014) Parameniscal cyst with associated extensive proximal tibial bone erosion: A case report and literature review. JBJS Case Connect 4(2): e46-e54.

13. Sobrino GC, Blazquez CMA, Astete CG, de la Puente Bujidos C (2015) Complicated parameniscal giant cyst with distal dissection and regional infection. Reumatol Clin 11(2): 116-117. 